\title{
ERRATUM
}

\section{Genetic susceptibility to invasive Salmonella disease}

James J. Gilchrist, Calman A. MacLennan and Adrian V. S. Hill

Nature Reviews Immunology 15, 452-463 (2015)

In the original version of this article, the credit line in the legend of Figure 1 was incorrect and should read: "Copyright 2014 from 'Vaccines against invasive Salmonella disease: current status and future directions' by MacLennan, C. A., Martin, L. B. \& Micoli, F.

Reproduced by permission of Taylor \& Francis LLC (http://www.tandfonline.com).”. This has now been corrected online. 